\section{Edema pulmonar agudo grave secundario a tratamiento de neumotórax espontáneo primario. Caso clínico}

\author{
CARLOS BARRIL MERINO ${ }^{1, a}$, MARÍA E. SOLOVERA R. ${ }^{1}$, \\ FELIPE BANNURA Y. ${ }^{1}$, PATRICIO SALAS V. ${ }^{1}$

\section{Pulmonary expansion edema during the management of a spontaneous pneumothorax. Report of one case}

Pulmonary expansion edema is a rare complication of the management of primary spontaneous pneumothorax. We report a 20 year old male admitted with a right primary spontaneous pneumothorax. A chest tube connected to a water seal was placed, achieving lung expansion. Immediately, the patient presented hypotension and a reduction in arterial oxygen saturation to $78 \%$. Non-invasive ventilation was started. A chest $X$ ray showed extensive right lung edema. The patient was managed with noradrenaline and albumin infusion with good response. Pulmonary edema resolved on day 3 but air leak was persistant so, the patient required surgery to excise apical bullae in the right lung. He was discharged during the following days in good condition.

(Rev Med Chile 2018; 146: 1343-1346)

Key words: General Surgery; Pneumothorax; Pulmonary Edema.
'Departamento de Cirugía,

Facultad de Medicina, Pontificia Universidad Católica de Chile.

Santiago, Chile.

aResidente Cirugía General,

Pontificia Universidad Católica de

Chile, Santiago, Chile.

Trabajo no recibió

financiamiento.

Los autores declaran no tener

conflictos de interés.

Recibido el 2 de febrero de 2018, aceptado el 18 de octubre de 2018.

Correspondencia a: Patricio Salas Villarroel psalas@med.puc.cl
$\mathrm{E}$ l edema pulmonar agudo por expansión es una entidad poco frecuente que se espera evitar en el manejo del neumotórax espontáneo, pero que rara vez se discute activamente cómo evitarlo o la última evidencia respecto al tratamiento. A continuación, se describe un caso clínico y una revisión del tema.

\section{Caso clínico}

Paciente de sexo masculino, 20 años de edad, sin antecedentes mórbidos relevantes, consulta por seis días de evolución de dolor torácico derecho de inicio súbito, de tope inspiratorio, con disnea. Radiografía muestra neumotórax derecho de $100 \%$. Ingresa por urgencias, se realiza pleurostomía con tubo $24 \mathrm{Fr}$, conectado a trampa de agua sin aspiración, logrando expansión pulmonar. Inmediatamente evoluciona con hipotensión hasta $90 / 60 \mathrm{mmHg}$, taquicárdico en promedio 120 latidos por minuto, saturando hasta $78 \%$ con $\mathrm{O}_{2}$ ambiental y sudoroso. Se optimiza la oxigenación con mascarilla de recirculación con oxígeno al $100 \%$ más nebulización con Fenoterol más Bromuro de Ipatropio alcanzando $92 \%$. Por lo anterior se decide traslado para monitorización en unidad de paciente crítico.

Ingresa a la unidad de intermedio con saturación hasta $88 \%$, por lo que se inicia apoyo ventilatorio mecánico no invasivo en modalidad CPAP. Radiografía de tórax portátil muestra extenso edema pulmonar derecho con falta de expansión apical y pulmón izquierdo sin signos de edema o condensación (Figura 1). A las $12 \mathrm{~h}$ de ingresado cursa con hipotensión, con requerimiento 


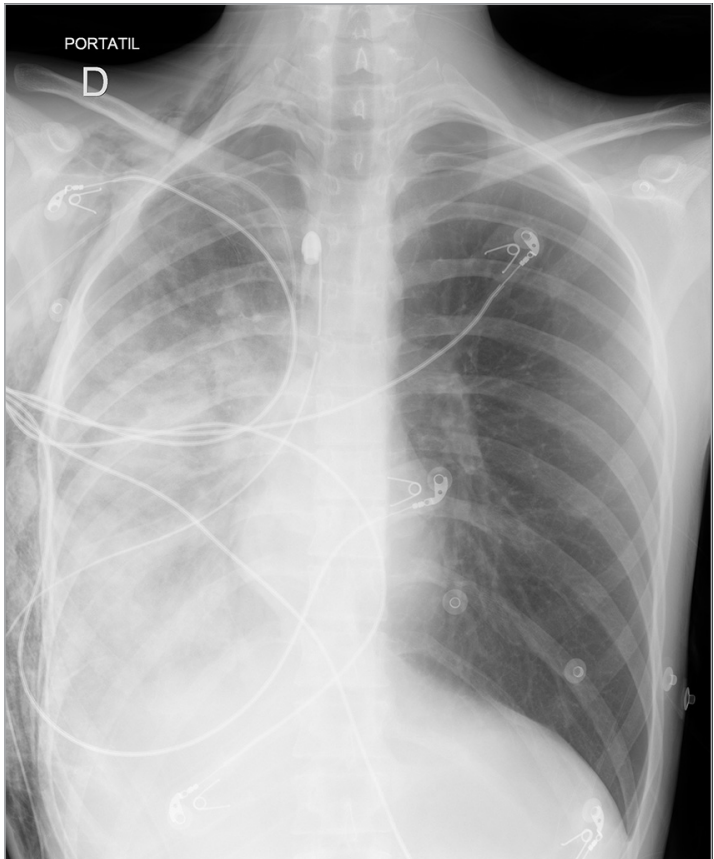

Figura 1. Radiografía portátil de tórax post inserción del tubo y de iniciado el cuadro de edema.

de noradrenalina para mejorar perfusión, en el contexto de shock probablemente distributivo se inicia aporte de volumen con suero ringer lactato e infusión de albúmina, con buena respuesta, no siendo necesarios drogas vasoactivas luego del primer día de su admisión.

A las $36 \mathrm{~h}$ de ingreso persiste con saturación de oxígeno cercana a $90 \%$ con $\mathrm{FiO}_{2} 30 \%$ por lo que se realiza AngioTAC de tórax que muestra opacidades en vidrio esmerilado compatible con edema por expansión en pulmón derecho y bulas apicales subpleurales bilaterales, de hasta $1,8 \mathrm{~cm}$ de tamaño a derecha y $4,8 \mathrm{~cm}$ a izquierda. Se decide mantener en manejo médico hasta mejorar parámetros ventilatorios para plantear resolución quirúrgica. Persiste con fuga aérea por el drenaje pleural y falta de expansión pulmonar por lo que se conecta a aspiración a $-6 \mathrm{cmH}_{2} \mathrm{O}$ bien tolerada.

Evoluciona estable, con requerimientos de oxígeno a la baja, siendo retiradas la ventilación mecánica no invasiva de forma progresiva, alcanzando cifras de saturación de $98 \%$ al quinto día sin apoyo ventilatorio suplementario. Pese a esto, persiste con fuga de aire y falta de expansión pulmonar. Al quinto día se controla con nueva radiografía

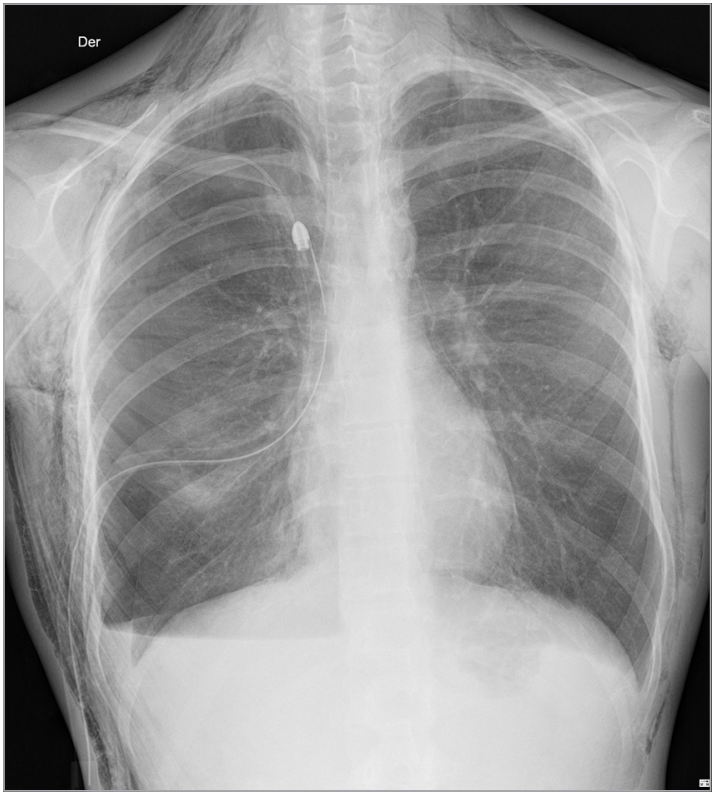

Figura 2. Radiografía portátil de tórax en el quinto día de manejo médico.

de tórax que muestra resolución del edema casi en su totalidad y falta de expansión de 50\% (Figura 2). Dada la resolución del edema se decide la cirugía. Se accede por videotoracoscopía derecha encontrando un pulmón de aspecto sano con un complejo de bulas apicales que se reseca mediante endograpadora. Además se realiza escarificación mecánica de la pleura. Al día siguiente de la cirugía evoluciona estable, con débito por pleurostomía serohemático, escaso, sin fuga de aire observable a la espiración forzada o maniobras de valsalva.

Se retira tubo pleural a las $48 \mathrm{~h}$ sin incidentes, radiografía de control con expansión completa.

\section{Discusión}

El edema pulmonar por expansión fue descrito por primera vez 1853 por Pinault en el contexto de toracocentesis y desde entonces se ha descrito como complicación aguda en el manejo del neumotórax espontáneo ${ }^{1}$.

Generalmente, se presenta posterior a la expansión del pulmón colapsado más de $72 \mathrm{~h}$ de presentación usualmente unilateral y rápida. La 
etiopatogenia es multifactorial: duración de los síntomas, cuantía del neumotórax y técnica de drenaje utilizada, entre otros ${ }^{2}$.

Distintas series de casos han descrito perfiles distintos de pacientes; En cuanto a incidencia, Yoon et al. describe una serie de 306 pacientes de los cuales 49 presentaron edema por expansión (16\%), todos con mínimo $56 \%$ de colapso vs $34 \%$ promedio de los pacientes que no presentaron esta complicación ${ }^{3}$, Haga et al. en una serie de 462 pacientes describe el cuadro en $30(6,5 \%)^{4}$ mientras que Matsuura en 14\% de 146 pacientes $^{5}$.

En 1959 Mahfood et al. en una serie de 47 casos evidenciaron la formación de edema en la primera hora en el $64 \%$ de los pacientes, todos desarrollaron edema a las $24 \mathrm{~h}$, de los cuales el 94\% tuvo edema ipsilateral. Con respecto al tiempo de espera, $83 \%$ había tenido clínica compatible con neumotórax por más de tres días, pero solo un $8 \%$ menos de una hora previa a la consulta ${ }^{6}$. La duración de la sintomatología previa a la consulta es un factor de riesgo clave, en la misma serie de Haga et al. los pacientes en promedio esperaron 7,7 días previo a la consulta versus 2,4 días de espera en los pacientes que no presentaron edema ${ }^{4}$.

Además del tiempo de espera, se presenta como factor el grado de colapso; en pacientes con menos de $30 \%$ no se desarrolla edema, versus el $17 \%$ que si desarrolla esta patología cuando el grado de colapso es mayor que la cifra indicada anteriormente ${ }^{5}$.

Tanto el tiempo de duración como el porcentaje de colapso encuentran su explicación en la patofisiología que hasta el minuto se tiene como consenso; la expansión brusca altera la producción de surfactante, a la vez que causa daño por reperfusión produciendo liberación de radicales libres e injuria microvascular con engrosamiento de la pared $^{7,8}$. Otro factor de riesgo a considerar es la diabetes mellitus, la cual se ha asociado a un daño microangiopático que altera la membrana basal produciendo depósito anómalo de colágeno tipo IV con el consecuente engrosamiento de ésta, lo que contribuye a generar una matriz donde el edema por reexpansión es más probable de ocurrir9.

El manejo del edema pulmonar por expansión es esencialmente de soporte; ventilación con aporte de oxígeno en modalidad CPAP en caso de que no se alcancen cifras de saturación adecuadas. Dependiendo del grado de cortocircuito pulmonar pudiera ser necesario el manejo con ventilación mecánica invasiva con presión de expiración positiva o $\mathrm{PEEP}^{10}$. El cortocircuito puede crear hipoxia y/o hipovolemia, que requiere manejo más activo con aporte de volumen, expansores de plasma e inotrópicos. Los diuréticos no son recomendables, ya que pueden empeorar la hipovolemia. La ventilación diferenciada es necesaria de forma excepcional ${ }^{11}$.

Cobra especial importancia la prevención de esta complicación. Para los pacientes que cumplan los factores de riesgo previamente expuestos (hombre joven, tiempo de duración, entre otros) se recomienda no dejar aspirativa la pleurostomía. Algunos grupos realizan punciones aspirativas en las que evitan extraer más de $1 \mathrm{~L}$ de aire cada vez (en otros casos se describe hasta $1.800 \mathrm{ml}$ como un volumen seguro). Considerando que la extracción fraccionada del aire, muchas veces en un paciente que además presenta fuga aérea, es un procedimiento complejo y poco estandarizado, es que se recomienda prestar especial atención a los síntomas de la formación inicial del edema, que son la tos persistente y el deterioro del estado general y ventilatorio ${ }^{14}$.

\section{Conclusión}

El edema pulmonar por expansión es una complicación grave del drenaje post neumotórax. La sospecha inicial está dada por la aparición de tos persistente asociado a compromiso hemodinámico y ventilatorio progresivo. El diagnóstico es por imagen y el manejo una vez diagnosticado consta de soporte ventilatorio acorde al grado de compromiso y hospitalización en una unidad monitorizada.

Se deben tener en cuenta los factores de riesgo para el desarrollo de esta condición, por lo cual las recomendaciones clásicas consisten en tratar de no extraer más del volumen recomendado, evitar la aspiración en la trampa de agua y mantener un alto índice de sospecha para poder iniciar el tratamiento de soporte de manera precoz.

\section{Referencias}

1. Verhagen M, van Buijtenen J, Geeraedts Jr L. Reexpansion pulmonary edema after chest drainage for pneumothorax: A case report and literature overview. Respiratory Medicine Case Reports 2015; 14: 10-2. 
2. Sherman S. Reexpansion pulmonary edema: a case report and review of the current literature. J Emerg Med 2013; 24: 23-4.

3. Yoon J, Suh J, Choi Y, Kwon J, Lee B, Lee S, et al. Risk factors for the development of reexpansion pulmonary edema in patients with spontaneous pneumothorax. Journal of Cardiothoracic Surgery 2013; 8: 164-4.

4. Haga T, Kurihara M, Kataoka H. The risk for re-expansion pulmonary edema following spontaneous pneumothorax. Surg Today 2014; 44: 1823-4.

5. Matsuura Y, Nomimura T, Murakami H, Matushima T, Kakehashi M, Kajihara H. Clinical analysis of reexpansion pulmonary edema. Chest 1991; 100 (6): 1562-6.

6. Mahfood S, Hix W, Aaron B, Blaes P, Watson D. Reexpansion pulmonary edema. Ann Thorac Surg 1988; 45 (3): 340-5.

7. Sewell R, Fewel J, Grover F, Arom K. Experimental evaluation of reexpansion pulmonary edema. Ann Thorac Surg 1978; 26: 126-6.

8. Sohara Y. Reexpansion pulmonary edema. Ann Thorac
Cardiovasc Surg 2008; 14 (4): 205-9.

9. Dias O, Teixeira L, Vargas F. Reexpansion pulmonary edema after therapeutic thoracentesis. Clinic 2010; 65 (12): 1387-9.

10. Cho S, Lee J, Kim M. New treatment method for reexpansion pulmonary edema: differential lung ventilation. Ann Thorac Surg 2005; 80: 1933-4.

11. Tarver RD, Broderick LS, Conces Jr DJ. Reexpansion pulmonary edema. J Thorac Imaging 1996; 11 (3): 19811.

12. Baumann M, Strange C, Heffner J, Light R, Kirby T, Klein J, et al. Management of spontaneous pneumothorax, an American college of chest physicians Delphi consensus statement. Chest 2001; 119 (2): 590-12.

13. Abunasser J, Brown R. Safety of large-volume thoracentesis. Conn Med 2010; 74 (1): 23-6.

14. Van Herendael HE, Van den Heuvel MC, Van Roey GW, Soetens FM. Re-expansielongoedeem bij een patient na behandeling van een pneumothorax. Ned Tijdschr Geneeskd 2006; 150 (5): 259-3. 